Original

\title{
Postprandial serum C-peptide to plasma glucose ratio as a predictor of subsequent insulin treatment in patients with type 2 diabetes
}

\author{
Yoshifumi Saisho' ${ }^{1)}$, Kinsei Kou ${ }^{1)}$, Kumiko Tanaka ${ }^{1)}$, Takayuki Abe ${ }^{2)}$, Hideaki Kurosawa ${ }^{1)}$, Akira Shimada ${ }^{3)}$, \\ Shu Meguro ${ }^{1)}$, Toshihide Kawai ${ }^{1)}$ and Hiroshi Itoh ${ }^{1)}$ \\ 1) Department of Internal Medicine, Keio University School of Medicine, Tokyo 160-8582, Japan \\ ${ }^{2)}$ Center for Clinical Research, Keio University School of Medicine, Tokyo 160-8582, Japan \\ ${ }^{3)}$ Department of Internal Medicine, Saiseikai Central Hospital, Tokyo 108-0073, Japan
}

\begin{abstract}
Type 2 diabetes is a progressive disease and most patients with type 2 diabetes eventually need insulin therapy. The objective of this study was to clarify C-peptide immunoreactivity (CPR), a marker of beta cell function, as a predictor of requirement for insulin therapy. We conducted a retrospective study of 579 consecutive subjects with type 2 diabetes who were admitted to our hospital from 2000 to 2007 and were able to be followed up for at least 6 months after discharge. Fasting and postprandial serum CPR and urinary CPR levels had been measured during admission. Information about insulin therapy at the last visit was obtained from medical records. At the last visit, 364 subjects (62.9\%) were treated with insulin. Mean interval between discharge and the last visit was $4.5 \pm 2.3$ years. Serum and urine CPR levels at baseline were significantly associated with insulin treatment at the last visit $(P<0.001$ for all). Among CPR values, postprandial serum CPR to plasma glucose ratio (CPR index) showed the greatest area under the receiver operating characteristic (ROC) curve for insulin therapy. Multivariate logistic regression analysis evaluating the effect of postprandial CPR index adjusted for other confounders showed consistent results with unadjusted results. In conclusion, beta cell dysfunction is significantly correlated with future insulin therapy in patients with type 2 diabetes. Our study indicates that among CPR measurements, postprandial CPR index is the best predictive marker for future insulin therapy.
\end{abstract}

Key words: Type 2 diabetes, Insulin, Beta cell function, C-peptide

TYPE 2 diabetes is characterized by beta cell dysfunction and insulin resistance[1]. Despite recent progress in the treatment of diabetes, abnormal glucose metabolism in patients with type 2 diabetes usually deteriorates gradually and insulin therapy is finally needed in many cases[2-4].

C-peptide is well known to be a marker of beta cell function[5-6]. C-peptide is split from insulin in the secretory granules in the beta cell and co-secreted with insulin. Since insulin, but not C-peptide, is extracted by the liver, serum C-peptide level reflects endogenous insulin secretion more directly than does serum insulin level. Moreover, C-peptide is capable of assessing beta

Received Dec. 20, 2010; Accepted Feb. 12, 2011 as K10E-399

Released online in J-STAGE as advance publication Mar.10, 2011

Correspondence to: Yoshifumi Saisho, M.D., Ph.D., Department of Internal Medicine, Keio University School of Medicine, 35 Shinanomachi, Shinjuku-ku, Tokyo 160-8582, Japan.

E-mail: saish@sc.itc.keio.ac.jp cell function even in patients under insulin therapy.

Recent studies reported that C-peptide immunoreactivity (CPR) adjusted by plasma glucose level, referred to as CPR index, predicted patients with type 2 diabetes who needed insulin therapy[7-8]. However, to our knowledge, no report has assessed postprandial CPR index as a predictor of insulin therapy. Although early insulin initiation has been reported to preserve beta cell function in patients with type 2 diabetes[9-11], patients often hesitate to accept insulin therapy mainly because of its daily injection. Thus, prediction of patients with type 2 diabetes who will need insulin therapy is important so as to introduce insulin therapy as early as possible.

Abbreviations: CPR, C-peptide immunoreactivity; JDS, Japan Diabetes Society; JNS, Japan Nephrology Society; eGFR, estimated glomerular filtration rate; ROC, receiver operating characteristic; TZD, thiazolidinedione; $\alpha$-GI, $\alpha$-glucosidase inhibitor; CAD, coronary artery disease; AUC, area under the curve; IRI, immunoreactive insulin; GAD, glutamic acid decarboxylase; IA-2, insulinoma-associated antigen-2 
Therefore, in this study we addressed the following questions using our data from a large population with detailed medical information on more than 500 patients: 1) What kind of CPR measurement is the best predictor of need for insulin therapy? 2) Is the association between the CPR measurement and insulin treatment robust when adjusting for other confounders?

\section{Research Design and Methods}

\section{Subjects}

We conducted screening in 689 consecutive patients with type 2 diabetes who were admitted to our hospital from 2000 to 2007. Most patients had been admitted to our hospital because of poor glycemic control and received basal-bolus insulin therapy during admission. We excluded 1) subjects positive for glutamic acid decarboxylase (GAD) or insulinoma-associated antigen-2 (IA-2) antibody, 2) subjects with serum creatinine level of $2.0 \mathrm{mg} / \mathrm{dL}$ or more, 3) subjects with fasting plasma glucose (FPG) of $200 \mathrm{mg} / \mathrm{dL}$ or more, and 4) subjects whose serum CPR level was not available in the medical records. Of the 689 patients, 110 patients (16.0\%) were not able to be followed up for at least 6 months mainly because of referral to another clinic or institution. Thus, 579 patients were eligible for analysis (Table 1). Mean interval between discharge and the last visit was $4.5 \pm 2.3$ years. Information about insulin therapy at the last visit was obtained from medical records in 2009. Insulin therapy was introduced based on clinical judgment according to the Japan Diabetes Society (JDS) guidelines for treatment of diabetes[12]. Briefly, if the optimal glycemic goal (HbA1c $<6.5 \%$, FPG $<130 \mathrm{mg} / \mathrm{dL}$ or $2 \mathrm{~h}$ postprandial plasma glucose $<180 \mathrm{mg} / \mathrm{dL}$ ) was not achieved by lifestyle modification and use of oral hypoglycemic agents, insulin therapy was considered. Lifestyle modification including diet therapy with ideal caloric intake calculated from ideal body weight (i.e., height $(\mathrm{m})^{2}$ x 22 x 25-30 kcal/ $\mathrm{kg}$ ) and moderate aerobic exercise at least $150 \mathrm{~min} /$ week was introduced at the first visit by doctors, nurses and nutritionists. The patients were also encouraged to participate in group counseling. Private counseling by nurses and nutritionists was further added if the optimal glycemic goal was not achieved. Especially, if optimal glycemic control was not achieved with the use of more than half the maximum dose of sulfonylureas, insulin therapy was considered. However, insulin therapy was also started regardless of prior treatment if the doctor considered that the patient needed insulin therapy. On the other hand, if optimal glycemic control was maintained with less than $10 \mathrm{U} /$ day of insulin, the doctor considered the possibility of discontinuing insulin therapy. This study was conducted according to the principles expressed in the Declaration of Helsinki and was approved by the ethics review committee of Keio University School of Medicine, Tokyo, Japan.

\section{Measurements}

All measurements were performed by the Department of Laboratory Medicine, Keio University School of Medicine with routine automated laboratory methods as previously reported[13]. HbA1c was measured by HPLC and expressed as international standard value; i.e. HbA1c (JDS) $+0.4 \%$, as defined by JDS[14]

Plasma glucose and serum CPR levels were measured after overnight fasting and $2 \mathrm{~h}$ after breakfast during admission, usually within a few days after admission under basal-bolus insulin therapy. All patients were receiving the ideal dietary calorie intake calculated from their ideal body weight (i.e. height $(\mathrm{m})^{2} \mathrm{x}$ $22 \times 25 \mathrm{kcal} / \mathrm{kg}$ ) when blood samples were obtained. Plasma glucose was measured by glucose oxidase method and CPR was measured by EIA. Coefficient of variance (CV) of the within-run and between-day precision of CPR was $2.39 \%$ and $2.97 \%$, respectively. Fasting and postprandial CPR indices were calculated as follows: fasting or postprandial serum CPR $(\mathrm{ng} / \mathrm{mL})$ / fasting or postprandial plasma glucose (mg/dL) x 100, respectively. In addition, $24 \mathrm{~h}$ urinary CPR was also measured. Urinary CPR index was calculated as $24 \mathrm{~h}$ urinary CPR ( $\mu$ g/day) / FPG (mg/dL).

Estimated glomerular filtration rate (eGFR: $\mathrm{mL} /$ $\min / 1.73 \mathrm{~m}^{2}$ ) was calculated according to the Statement of the Japan Nephrology Society (JNS)[15-16] as follows: $194 \mathrm{x}$ serum creatinine $(\mathrm{mg} / \mathrm{dL})^{-0.194} \mathrm{x}$ age (years) ${ }^{-0.287}$ (x 0.739 for women).

Diabetic complications were precisely evaluated during admission. Diagnosis of diabetic retinopathy was performed by ophthalmologists.

\section{Statistical analysis}

Descriptive statistics were calculated for the baseline characteristics. Homogeneity of distributions between the two groups was examined with MannWhitney's U test or Fisher's exact test. Association between CPR level at discharge and insulin treatment at the last visit was evaluated with a logistic regression 
Table 1 Baseline characteristics of subjects according to insulin therapy at last visit.

\begin{tabular}{|c|c|c|c|}
\hline & Total & Insulin therapy (-) & Insulin therapy $(+)$ \\
\hline $\mathrm{N}$ & 579 & 215 & 364 \\
\hline Male (\%) & 62.7 & 64.2 & 61.8 \\
\hline Age (years) & $63 \pm 12$ & $63 \pm 13$ & $63 \pm 12$ \\
\hline Duration of diabetes (years) & $13 \pm 10$ & $9 \pm 9$ & $15 \pm 10 \#$ \\
\hline Duration between discharge and last visit (years) & $4.5 \pm 2.3$ & $4.2 \pm 2.2$ & $4.7 \pm 2.3^{*}$ \\
\hline Family history of diabetes (\%) & 49.9 & 43.3 & $53.9 *$ \\
\hline BMI $\left(\mathrm{kg} / \mathrm{m}^{2}\right)$ & $24.4 \pm 4.1$ & $24.9 \pm 4.2$ & $24.1 \pm 4.0^{*}$ \\
\hline HbA1c (\%) & $9.9 \pm 1.8$ & $9.9 \pm 2.0$ & $9.9 \pm 1.7$ \\
\hline eGFR (mL/min) & $70.4 \pm 24.7$ & $69.5 \pm 21.5$ & $70.9 \pm 26.5$ \\
\hline \multicolumn{4}{|l|}{ Medication before admission } \\
\hline Sulfonylurea (\%) & 49.2 & 39.1 & $55.2 \#$ \\
\hline Glinide (\%) & 1.9 & 3.3 & 1.1 \\
\hline Biguanide (\%) & 16.4 & 10.3 & $17.6^{*}$ \\
\hline TZD (\%) & 7.8 & 4.2 & $9.9 * *$ \\
\hline$\alpha-G I(\%)$ & 27.8 & 19.1 & 33.0\# \\
\hline Insulin (\%) & 17.6 & 2.3 & $26.7 \#$ \\
\hline \multicolumn{4}{|l|}{ Complications } \\
\hline Diabetic retinopathy (\%) & 39.7 & 22.3 & $50.0 \#$ \\
\hline Diabetic nephropathy (\%) & 38.9 & 29.8 & $44.2 \#$ \\
\hline Diabetic neuropathy (\%) & 57.3 & 45.1 & $64.6 \#$ \\
\hline CAD (\%) & 19.7 & 14.4 & $22.8 *$ \\
\hline Stroke (\%) & 14.5 & 11.6 & 16.2 \\
\hline \multicolumn{4}{|l|}{ CPR indices } \\
\hline FPG (mg/dL) & $143 \pm 30$ & $142 \pm 29$ & $144 \pm 31$ \\
\hline Fasting CPR (ng/mL) & $1.75 \pm 1.03$ & $2.08 \pm 0.99$ & $1.56 \pm 1.00 \#$ \\
\hline Fasting CPR index & $1.25 \pm 0.75$ & $1.51 \pm 0.73$ & $1.10 \pm 0.71 \#$ \\
\hline Postprandial PG (mg/dL) & $235 \pm 62$ & $223 \pm 59$ & $242 \pm 63 \#$ \\
\hline Postprandial CPR (ng/mL) & $4.34 \pm 2.58$ & $5.61 \pm 2.75$ & $3.59 \pm 2.14 \#$ \\
\hline Postprandial CPR index & $1.96 \pm 1.35$ & $2.70 \pm 1.61$ & $1.52 \pm 0.92 \#$ \\
\hline Urinary CPR ( $\mu \mathrm{g} /$ day) & $64.0 \pm 51.3$ & $78.8 \pm 57.3$ & $55.1 \pm 45.1 \#$ \\
\hline Urinary CPR index & $0.45 \pm 0.36$ & $0.57 \pm 0.39$ & $0.39 \pm 0.31 \#$ \\
\hline
\end{tabular}

eGFR; estimated glomerular filtration rate, FPG; fasting plasma glucose, CPR; C-peptide immunoreactivity, TZD; thiazolidinedione, $\alpha$-GI; $\alpha$-glucosidase inhibitor, CAD; coronary artery disease. ${ }^{*} p<0.05,{ }^{* *} p<0.01, \# p<0.001$ vs. subjects not treated with insulin after discharge.

model for each CPR parameter. Correlations between two CPR parameters were estimated with Spearman's rank correlation coefficient. Receiver operating characteristic (ROC) analysis was performed to compare the performance of the predictors of insulin therapy. Multivariate logistic regression analysis was used to evaluate the effect of the postprandial CPR index on insulin treatment adjusted for other confounders. All statistical analyses were conducted using the Statistical Package for the Social Sciences (version 17.0; SPSS, Chicago, IL, USA). All data are expressed as mean \pm $\mathrm{SD}$, and values of $p<0.05$ (two-sided) were considered statistically significant.

\section{Results}

\section{Baseline characteristics of subjects according to insu- lin therapy at last visit}

Among 579 subjects, 364 subjects (62.9\%) were being treated with insulin at their last visit at $4.5 \pm 2.3$ years since discharge. In subjects treated with insulin, mean daily insulin dose was $0.42 \pm 0.24 \mathrm{U} / \mathrm{kg}$ (27 $\pm 18 \mathrm{U}$, range 2-102 $\mathrm{U}$ ), and 337 subjects (92.6\%) were treated with insulin injection twice a day or more. Among them, 325 subjects (89.3\%) had already commenced insulin therapy at the time of discharge. On the other hand, 57 subjects who were being treated with insulin at the time of discharge had been able to discon- 
Table 2 Association between baseline serum and urinary CPR levels and insulin therapy at last visit by individual CPR measurement.

\begin{tabular}{lcc}
\hline Variables & Adjusted odds ratio (95\% CI) & $p$ value \\
\hline Fasting CPR (ng/mL) & $0.60(0.50-0.71)$ & $<0.001$ \\
Fasting CPR index & $0.46(0.35-0.59)$ & $<0.001$ \\
Postprandial CPR (ng/mL) & $0.70(0.64-0.76)$ & $<0.001$ \\
Postprandial CPR index & $0.40(0.33-0.49)$ & $<0.001$ \\
Urinary CPR ( $\mu$ g/day) & $0.99(0.99-0.99)$ & $<0.001$ \\
Urinary CPR index & $0.22(0.13-0.38)$ & $<0.001$ \\
\hline
\end{tabular}

FPG; fasting plasma glucose, CPR; C-peptide immunoreactivity. A univariate logistic regression model was used for analysis.

Table 3 Correlations among markers of beta cell function.

\begin{tabular}{lcccccc}
\hline & FCPR & FCPRI & PCPR & PCPRI & uCPR & uCPRI \\
\hline Fasting CPR & - & 0.913 & 0.761 & 0.726 & 0.456 & 0.414 \\
Fasting CPR index & 0.913 & - & 0.717 & 0.786 & 0.380 & 0.433 \\
Postprandial CPR & 0.761 & 0.717 & - & 0.886 & 0.467 & 0.447 \\
Postprandial CPR index & 0.726 & 0.786 & 0.886 & - & 0.388 & 0.431 \\
Urinary CPR & 0.456 & 0.380 & 0.467 & 0.388 & - & 0.959 \\
Urinary CPR index & 0.414 & 0.433 & 0.447 & 0.431 & 0.959 & - \\
\hline
\end{tabular}

FCPR; fasting CPR, FCPRI; fasting CPR index, PCPR; postprandial CPR, PCPRI; postprandial CPR index, uCPR; urinary CPR, uCPRI; urinary CPR index. All $p<0.001$.

tinue insulin therapy at the last visit. Demographic factors and baseline characteristics for the two groups are summarized in Table 1. Patients who were treated with insulin at the last visit showed significantly longer duration of diabetes $(p<0.001)$ and lower BMI $(p<0.05)$, but comparable HbA1c level $(p=0.91)$ at the time of admission compared with those without insulin therapy. Patients who were treated with insulin at the last visit also showed a significantly higher proportion who had used oral hypoglycemic agents or insulin previously. The incidence of diabetic complications except stroke was also significantly higher in patients with insulin therapy compared with those without insulin.

\section{Baseline CPR indices of subjects according to insulin therapy at last visit}

Patients who were being treated with insulin at the last visit showed significantly lower fasting and postprandial CPR levels compared with those without insulin, while postprandial plasma glucose level was significantly higher in those with insulin (all $p<0.001$ ) (Table 1). As a result, fasting and postprandial CPR indices were significantly lower in patients with insulin therapy compared to those without insulin $(p<0.001)$.
Urinary CPR and urinary CPR index were both significantly lower in those with insulin therapy $(p<0.001)$.

\section{Association between baseline CPR level and insulin treatment at last visit}

The association between baseline CPR level and insulin treatment at the last visit was evaluated with a logistic regression model for individual parameters (Table 2). Each CPR parameter showed significant association with insulin treatment at the last visit ( $p$ $<0.001$ for all).

\section{Correlation and predictive performance of CPR indices}

Fasting and postprandial serum CPR levels were strongly correlated with each other in terms of both absolute values and indices (Table 3). While urinary CPR and urinary CPR index were strongly correlated with each other, they showed only modest correlations with each serum CPR index (Table 3).

To estimate the predictive performance for insulin therapy among these CPR indices, we performed ROC analysis. ROC analysis for insulin therapy showed the greatest point estimate of the area under the curve (AUC) for postprandial CPR index $(0.779,95 \% \mathrm{CI}$ : 
0.741-0.818) compared to the other markers (Figure 1), suggesting that postprandial CPR index is the best predictor of insulin therapy among the CPR indices. The best cut-off value of postprandial CPR index for predicting insulin therapy was 2.02 , with $80.8 \%$ sensitivity and $63.3 \%$ specificity. If specificity was increased to $80.5 \%$, the cut-off value of postprandial CPR index became 1.53 , with $61.0 \%$ sensitivity. Similar results were obtained when the patients who were already using insulin at the time of admission $(n=102)$ were excluded from the analysis (AUC for postprandial CPR index: 0.769, 95\% CI: 0.727-0.811, fasting CPR index: 0.685, 95\% CI: 0.638-0.733, urinary CPR index: 0.601, 95\% CI: 0.549-0.653).

\section{Sensitivity analyses}

Finally, to evaluate the robustness of the unadjusted association between the CPR measurement and insulin treatment, we performed multivariate logistic analysis including sex, age, duration of diabetes, family history of diabetes, BMI, HbA1c, eGFR, postprandial CPR index, previous medication (sulfonylurea, glinide, biguanide, thiazolidinedione, $\alpha$-glucosidase inhibitor or insulin), and presence of diabetic complications (diabetic retinopathy, diabetic nephropathy, diabetic neuropathy, coronary artery disease (CAD) and stroke) as independent variables. As a result, postprandial CPR index, pretreatment with insulin, pretreatment with sulfonylurea, diabetic retinopathy, age, CAD and diabetic neuropathy were selected as independent predictors of insulin therapy (Table 4). The adjusted odds ratio of postprandial CPR index was 0.45 (95\% CI: 0.37-0.56) for each 1 increment of the index, consistent with the result from the unadjusted model.

In this study, 41 patients who were treated with insulin at the last visit used less than $10 \mathrm{U} /$ day of insulin.

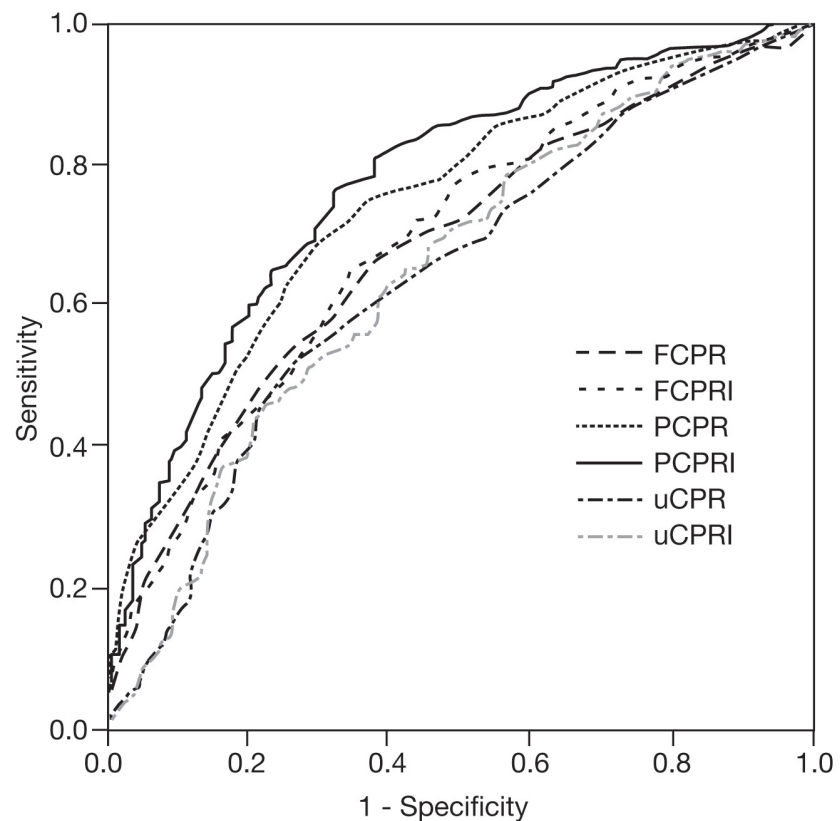

Fig. 1 Receiver-operating characteristic (ROC) curves of markers of beta cell function for insulin treatment. Postprandial CPR index showed the greatest area under the curve (AUC) of 0.779 (95\% CI: 0.741-0.818) compared to the other markers. AUCs (95\% CI) of other variables were as follows: FCPR; 0.686 (0.642-0.730), FCPRI; 0.695(0.6510.738), PCPR; 0.747 (0.707-0.788), uCPR; 0.641 (0.5940.688), and uCPRI; 0.650 (0.603-0.698). FCPR; fasting CPR, FCPRI; fasting CPR index, PCPR; postprandial CPR, PCPRI; postprandial CPR index, uCPR; urinary CPR, uCPRI; urinary CPR index.

We also confirmed the same results in insulin-treated patients who used less than $10 \mathrm{U} /$ day of insulin at the last visit as those in the non-insulin-treated group (ROC-AUC and odds ratio of postprandial CPR index: 0.746 (95\% CI: 0.706-0.787) and 0.47 (0.38-0.58), respectively).

Table 4 Multivariate logistic regression analysis for insulin treatment.

\begin{tabular}{lcc}
\hline Variables & Adjusted odds ratio (95\%CI) & $p$ value \\
\hline Postprandial CPR index & $0.45(0.37-0.56)$ & $<0.001$ \\
Pretreatment with insulin & $18.99(6.50-55.49)$ & $<0.001$ \\
Pretreatment with sulfonylurea & $2.86(1.84-4.47)$ & $<0.001$ \\
Diabetic retinopathy & $2.00(1.26-3.19)$ & 0.003 \\
Age (years) & $0.97(0.96-0.99)$ & 0.004 \\
CAD & $1.89(1.09-3.28)$ & 0.024 \\
Diabetic neuropathy & $1.57(1.01-2.42)$ & 0.042 \\
\hline
\end{tabular}

CAD; coronary artery disease. 


\section{Discussion}

In this study, 1) patients who subsequently needed insulin therapy showed lower CPR indices, 2) among the various CPR indices, postprandial CPR index was the best predictor of insulin therapy, and 3) the association between the postprandial CPR index and insulin treatment was robust and not dependent on the analysis model. Type 2 diabetes is a progressive disease, and beta cell function gradually decreases with the duration of type 2 diabetes[2-4]. It has been reported that early insulin treatment may prevent the decline of beta cell function[9-10]. Therefore, it is important to identify predictors of the need for insulin therapy in order to introduce early insulin treatment for patients with type 2 diabetes who need it.

Recent studies have reported that fasting CPR index and urinary CPR both predict insulin therapy[7-8]. Their findings were in line with our findings, while we further compared the indices including postprandial state and found that the postprandial CPR index was a superior predictor compared with either fasting CPR index or urinary CPR index. We also confirmed that the CPR index was a superior predictor of insulin therapy compared to serum CPR level itself in both a fasting and a postprandial state in our larger population including more than 500 patients. Since plasma glucose stimulates insulin secretion, it is not surprising that a CPR index, i.e., serum CPR level adjusted by plasma glucose level, reflects endogenous insulin secretion ability more precisely than does serum CPR level itself. In the postprandial state, insulin secretion is further stimulated by both a higher plasma glucose level and incretin action[17]. Thus, postprandial CPR index may be more useful to assess beta cell functional capacity compared to fasting CPR index.

On the other hand, both urinary CPR and urinary CPR index showed only modest correlations with serum CPR indices and a smaller AUC of ROC analysis for insulin therapy compared to serum CPR indices. The reason for this could be sampling error (insufficient urine storage) or the presence of subclinical urinary tract infection, which would affect CPR measurement. Moreover, since urinary CPR reflects daily total insulin secretion, adjustment for only fasting plasma glucose level may not be sufficient to adjust for daily total insulin secretion, especially considering the postprandial state. Unfortunately, since we did not measure plasma insulin levels (immunoreactive insulin, IRI) in this study, we were not able to compare the usefulness of CPR index with that of IRI.

ROC analysis demonstrated that if the cut-off value of postprandial CPR index is set at 1.5 , the specificity for insulin therapy would be $80 \%$ (60\% sensitivity). Moreover, multivariate logistic regression analysis evaluating the effect of the postprandial CPR index adjusted for other confounders showed consistent results, and the estimated odds ratio of 1 unit of the postprandial CPR index was 0.45 .

Logistic regression analysis also revealed other factors predicting insulin therapy such as previous treatment with insulin, previous treatment with sulfonylureas, diabetic retinopathy, age, CAD and diabetic neuropathy, but not HbA1c, suggesting that baseline HbA1c level may not predict insulin therapy. Unexpectedly, the duration of diabetes was not selected in the analysis. The reason for this may be 1) in the multivariate analysis, the effect of the duration of diabetes was explained by other variables that correlated with it, or 2) the difficulty in exactly estimating the time of onset of type 2 diabetes. It should be noted that the clinical decision to introduce insulin therapy during admission itself could be influenced by CPR indices as well as the presence of diabetic complications. To reduce this possibility, we assessed the use of insulin at the last visit, which was $4.5 \pm 2.3$ years after discharge. Moreover, patients with insulin therapy at the last visit were receiving a total daily dose of $0.42 \pm 0.24 \mathrm{U} / \mathrm{kg}$ of insulin and the majority injected twice a day or more, suggesting their need for insulin. Nonetheless, the mean $\mathrm{HbA1c}$ level at the last visit was rather higher in subjects with insulin therapy than in those without (7.4 \pm 1.4 vs. $6.7 \pm 1.2, p<0.001)$, also suggesting that our clinical judgment to introduce insulin had been reasonable. This finding also indicates that postprandial CPR index may be a useful predictor of future deterioration of glucose tolerance. We also confirmed the same results in insulin-treated patients who used less than 10 $\mathrm{U} /$ day of insulin at the last visit $(\mathrm{n}=41)$ as in the noninsulin-treated group.

There are several limitations of our study. First, we used logistic regression analysis rather than a Cox proportional-hazards model to identify independent variables predicting insulin therapy, because we did not have information on the time of insulin initiation. However, since $~ 90 \%$ of the subjects who were treated with insulin at the last visit had already commenced insulin therapy at the time of discharge, the difference 
should be minimal. Second, we excluded subjects with renal failure, which affects CPR measurement. Thus, the results may not be applicable to subjects with renal failure. Third, we also excluded subjects whose FPG was $200 \mathrm{mg} / \mathrm{dL}$ or more in order to exclude the possibility that beta cell function was transiently impaired by marked hyperglycemia. Since hyperglycemia per se could affect beta cell function, which is generally recognized as glucose toxicity, CPR may not reflect true beta cell function when marked hyperglycemia is present. Nonetheless, because of this, our results may not be applicable to subjects with marked hyperglycemia. Finally, because of the retrospective design of this study, the patients who were treated with insulin at the last visit had a longer follow-up duration between discharge and the last visit compared to those without insulin (Table 1). Therefore, we also performed subgroup analysis stratified by the duration between discharge and last visit of 4 years or less, or more than 4 years ( $n=313$ and 266, respectively). As a result, there was no significant difference in the follow-up duration between the patients treated with or without insulin in both subgroups ( $2.6 \pm 1.1$ vs. $2.7 \pm 1.1$ years, $p=0.46$ and $6.5 \pm 1.3$ vs. $6.7 \pm 1.4$ years, $p=0.34$ for subgroups with follow-up duration of 4 years or less and more than 4 years, respectively). In this subgroup analysis, we confirmed that the results were not changed in either subgroup (ROC-AUC of postprandial CPR index: 0.799 (95\%CI: 0.749-0.848) and 0.758 (0.6980.818 ), odds ratio of postprandial CPR index: 0.32 (0.24-0.44) and 0.49 (0.38-0.64) for subgroups with follow-up duration of 4 years or less and more than 4 years, respectively).

In conclusion, beta cell dysfunction is significantly correlated with future insulin therapy in patients with type 2 diabetes. Among CPR indices, postprandial CPR index is the best predictive marker of future insulin therapy. Assessing postprandial CPR index may be useful for starting insulin therapy as early as possible in patients with type 2 diabetes in order to preserve their beta cell function.

\section{Acknowledgements}

The authors have no conflict of interest. The authors thank Dr. Wendy Gray for editing the manuscript.

\section{References}

1. Defronzo RA (2009) Banting Lecture. From the triumvirate to the ominous octet: a new paradigm for the treatment of type 2 diabetes mellitus. Diabetes 58: 773795.

2. Matthews DR, Cull CA, Stratton IM, Holman RR, Turner RC (1998) UKPDS 26: Sulphonylurea failure in non-insulin-dependent diabetic patients over six years. UK Prospective Diabetes Study (UKPDS) Group. Diabet Med 15: 297-303.

3. Festa A, Williams K, D’Agostino R, Jr., Wagenknecht LE, Haffner SM (2006) The natural course of beta-cell function in nondiabetic and diabetic individuals: the Insulin Resistance Atherosclerosis Study. Diabetes 55: 1114-1120.

4. Funakoshi S, Fujimoto S, Hamasaki A, Fujiwara H, Fujita Y, Ikeda K, Hamamoto Y, Hosokawa M, Seino Y, Inagaki N (2008) Analysis of factors influencing pancreatic beta-cell function in Japanese patients with type 2 diabetes: association with body mass index and duration of diabetic exposure. Diabetes Res Clin Pract 82: 353-358.

5. Tsai EB, Sherry NA, Palmer JP, Herold KC (2006) The rise and fall of insulin secretion in type 1 diabetes mellitus. Diabetologia 49: 261-270.
6. Torn C (2003) C-peptide and autoimmune markers in diabetes. Clin Lab 49: 1-10.

7. Asano T, Kawamura M, Watanabe T, Abe M, Chin R, Miyazaki S, Hirata Y (2008) Indices of urinary and serum c-peptide corrected with fasting plasma glucose for decision-making of insulin therapy in type 2 diabetes-validation and comparison. J Japan Diab Soc 51: 759-763 (In Japanese).

8. Goto A, Takaichi M, Kishimoto M, Takahashi Y, Kajio H, Shimbo T, Noda M (2010) Body mass index, fasting plasma glucose levels, and C-peptide levels as predictors of the future insulin use in Japanese type 2 diabetic patients. Endocr J 57: 237-244.

9. Weng J, Li Y, Xu W, Shi L, Zhang Q, Zhu D, Hu Y, Zhou Z, Yan X, Tian H, Ran X, Luo Z, Xian J, Yan L, Li F, Zeng L, Chen Y, Yang L, Yan S, Liu J, Li M, Fu Z, Cheng H (2008) Effect of intensive insulin therapy on beta-cell function and glycaemic control in patients with newly diagnosed type 2 diabetes: a multicentre randomised parallel-group trial. Lancet 371: 1753-1760.

10. Alvarsson M, Sundkvist G, Lager I, Berntorp K, Fernqvist-Forbes E, Steen L, Orn T, Holberg MA, Kirksaether N, Grill V (2008) Effects of insulin vs. glibenclamide in recently diagnosed patients with type 2 
diabetes: a 4-year follow-up. Diabetes Obes Metab 10: 421-429.

11. Chen HS, Wu TE, Jap TS, Hsiao LC, Lee SH, Lin HD (2008) Beneficial effects of insulin on glycemic control and beta-cell function in newly diagnosed type 2 diabetes with severe hyperglycemia after short-term intensive insulin therapy. Diabetes Care 31: 1927-1932.

12. The Japan Diabetes Society (2004) Evidence-based practice guideline for the treatment of diabetes in Japan. Nankodo, Tokyo (In Japanese).

13. Saisho Y, Miyakoshi K, Tanaka M, Shimada A, Ikenoue S, Kadohira I, Yoshimura Y, Itoh H (2010) Beta cell dysfunction and its clinical significance in gestational diabetes. Endocr J 57: 973-980.

14. Seino Y, Nanjo K, Tajima N, Kadowaki T, Kashiwagi A, Araki E, Ito C, Inagaki N, Iwamoto Y, Kasuga M, Hanafusa M, Haneda M, Ueki K (2010) Report of the
Committee on the Classification and Diagnostic Criteria of Diabetes Mellitus. J Japan Diab Soc 53: 450-467 (In Japanese).

15. Matsuo S, Imai E, Horio M, Yasuda Y, Tomita K, Nitta K, Yamagata K, Tomino Y, Yokoyama H, Hishida A (2009) Revised equations for estimated GFR from serum creatinine in Japan. Am J Kidney Dis 53: 982992.

16. Horio M, Imai E, Yasuda Y, Watanabe T, Matsuo S (2010) Modification of the CKD epidemiology collaboration (CKD-EPI) equation for Japanese: accuracy and use for population estimates. Am J Kidney Dis 56: 32-38.

17. Drucker DJ, Nauck MA (2006) The incretin system: glucagon-like peptide-1 receptor agonists and dipeptidyl peptidase-4 inhibitors in type 2 diabetes. Lancet 368: 1696-1705. 